\title{
Anti-K-RAS G12D mTCR-transduced Autologous Peripheral Blood Lymphocytes
}

National Cancer Institute

\section{Source}

National Cancer Institute. Anti-K-RAS G12D mT CR-transduced Autologous Peripheral

Blood Lymphocytes. NCI Thesaurus. Code C156889.

Autologous peripheral blood lymphocytes (PBLs) transduced with a retroviral vector encoding for an HLA class I histocompatibility antigen A*11:01 (HLA-A1101)-restricted murine T-cell receptor (mT CR) that recognizes the glycine (Gly, G) to aspartic acid (Asp, D) point mutation at position 12 (G12D) variant of K-RAS (KRAS), with potential immunomodulating and antineoplastic activities. HLA-A1101-positive PBLs are harvested from a K-RAS G12D-expressing cancer patient and transfected with a retroviral vector that encodes anti-K-RAS G12D mTCR. The transduced PBLs are then expanded in culture. When reintroduced to the patient, these anti-K-RAS G12D mTCR-expressing PBLs target and bind to K-RAS G12D-overexpressing tumor cells, which results in both cytokine secretion, including interferon-gamma (IFN-g), and tumor cell lysis. K-RAS, a member of the RAS family of oncogenes, serves an important role in cell signaling, division and differentiation. Mutation of K-RAS may induce constitutive signal transduction leading to tumor cell growth, proliferation, invasion, and metastasis. 